\title{
A Novel Reactor for Determination of Kinetics for Solid Catalyzed Gas Reactions
}

\author{
P. C. Borman, A. N. R. Bos, and K. R. Westerterp \\ Chemical Reaction Engineering Lab., Dept of Chemical Engineering, \\ University of Twente, 7500 AE Enschede, The Netherlands
}

\begin{abstract}
A novel perfectly mixed laboratory reactor for determining kinetics of heterogeneously catalyzed gas-phase reactions has been developed. Perfect mixing is achieved by circulating the gas in the reactor using an axial flow impeller in a well streamlined enclosure. Pellets are fixed in a rectangular opening in the blades of the impeller. They rotate with the impeller, thus realizing high particle velocities in the reactor. Interparticle mass transfer was studied experimentally by vaporization of naphthalene pellets. The mass-transfer coefficient in the novel reactor was found to depend on the velocity of a particle in the reactor. Mass-transfer coefficients in an internal recycle reactor at equal impeller tip speeds are 4-6 times lower than those in the novel reactor, and conditions can be chosen easily where at higher rotational speeds the mass- and heat-transfer rates are 8-10 times higher than in classical recycle reactors. The recycle flow rate in a recycle reactor was found to depend strongly on the resistance to flow caused by the catalyst bed itself. The novel reactor was tested under reacting conditions using the hydrogenation of ethene.
\end{abstract}

\section{Introduction}

Kinetic investigations are important for the design of largescale reactors. They are usually performed in special laboratory reactors. These have received much attention in literature, see, for example, Carberry (1964), Mahoney (1974), Berty (1974, 1979, 1984), Weekman (1974), Doraiswamy and Tajbl (1974), and Christoffel (1982). In kinetic experiments, the measured reaction rates should not be disguised by mass- and/or heattransfer resistances; moreover, temperatures and concentrations at which the reactions take place must be known accurately. In a perfectly mixed reactor, concentrations and temperature are uniform and can be determined easily. The interpretation of experimental data is simple in a gradientless reactor as no temperature and concentration profiles have to be interpreted like in an integral reactor type. A high gas velocity around the particles is required to avoid interparticle transport limitations.

In the spinning basket reactor, proposed by Carberry (1964), the catalyst particles are placed in a basket which rotates in

Current address of P. C. Borman: DSM Research, P.O. Box 18,6160 MD Geteen, The Netherlands.

Current address of A. N. R. Bos: Koninklijke/Shell-Laboratorium, Amsterdam, P.O. Box 3003,1003 AA Amsterdam, The Netherlands. an enclosure. It is assumed that the rotation of the basket ensures perfectly mixed conditions and high interparticle transport rates. In this reactor, the flow pattern of the gas around the particles is unknown, because the gas tends to spin along with the basket.

Another way to approach perfect mixed conditions is by using a packed bed reactor with a recycle of the effluent gas stream (Westerterp et al., 1987). In a recycle reactor, the conversion per pass over the catalyst bed can be kept small whereas the overall conversion over the reactor can be large. These large conversions facilitate reaction rate determinations as the demands on the accuracy of the analytical techniques are less stringent compared to differential operation. The first reactor design with an internal recycle was presented by Berty (1974). The reactor contains a basket in which a variable amount of catalyst can be placed and a magnetically driven blower for the internal gas circulation in the reactor. Over the years many alterations to the first design have been introduced, see Berty (1984). A number of problems with this type of reactor have been identified. Whether the reactor can be assumed to behave as perfectly mixed with temperature and concentration gradients having a negligible influence on the experimental re- 


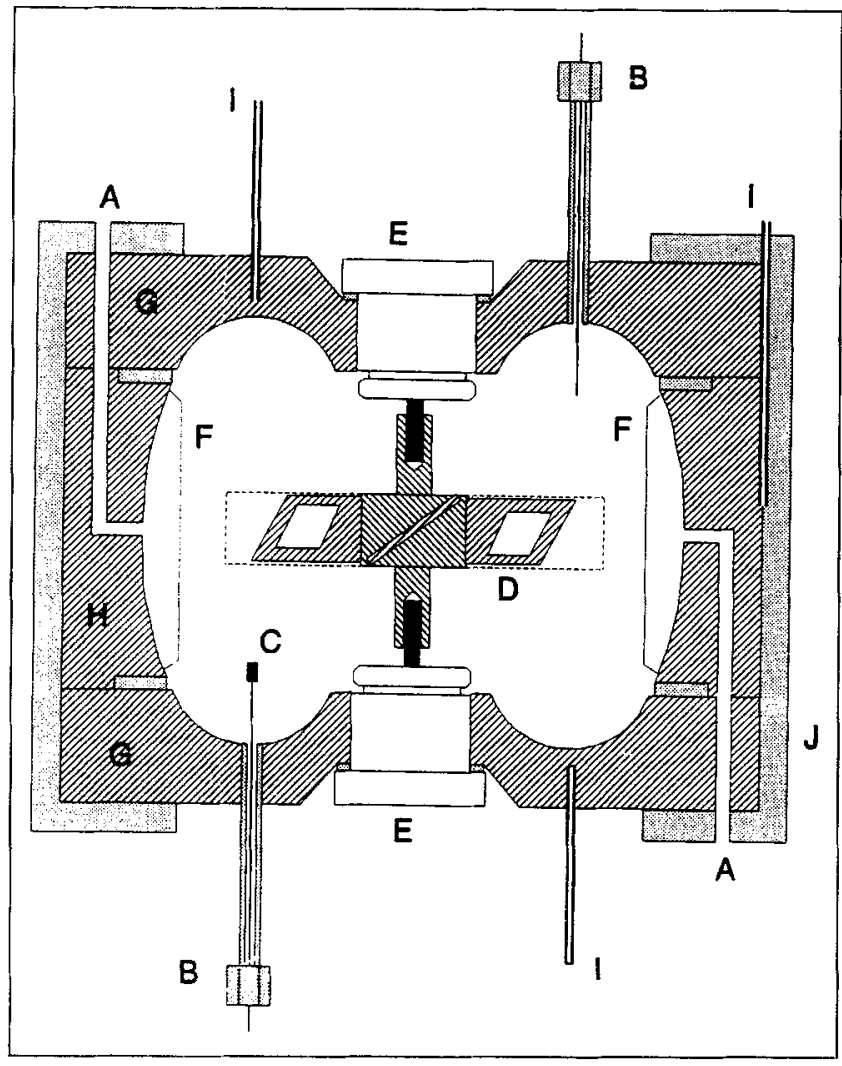

Figure 1. Novel reactor.

(A) gas in/outlet; (B) pressure sealed leads for thermocouples; (C) particle fixed on thermocouple; (D) axial flow impeller (Figure 2); (E) impeller bearing; (F) baffles; $(G)$ top/bottom part; $(\mathrm{H})$ center part; (I) thermowell; (J) bronze jacket. For dimensions see Table 1.

action rates, depends on the recycle and the feed flow rates, the total conversion over the reactor, the reaction kinetics, and the heat of reaction (Borman, 1992). The recycle flow rate is determined by the discharge rate of the blower. Unfortunately, this rate is hard to determine, so in practice it is difficult to prove whether perfect mixing has been achieved. The falsification of kinetic data, as well as procedures for correcting data obtained in reactors with a low recycle ratio, have been discussed (Pirjamali et al., 1973; Broucek, 1983; Georgakopoulos and Broucek, 1987; Wedel and Villadsen, 1983; Dümpelmann and Baiker, 1992; Borman, 1992). Generally, a low recycle ratio forces the experimentalist to restrict the overall conversion over the reactor. The interparticle heat- and mass-transfer rates in recycle reactors also depend on the recycle flow rate. A too low recycle flow rate can cause disguise of the measured reaction rates both by imperfect mixing as well as by transport resistances. Examples of both types of disguise of experimental data are discussed by Bos et al. (1992). Another practical problem with internal recycle reactors is the mechanical instability of the bearings of the impeller shaft. With the design of Berty (1974), the impeller only has a bearing on one side, and therefore it may wear out rapidly due to imbalances.

In this article, we introduce a novel design for a laboratory reactor in which recycle ratio problems have been solved, for fast reactions higher heat- and mass-transfer rates can be reached, and mechanical problems are not exhibited.

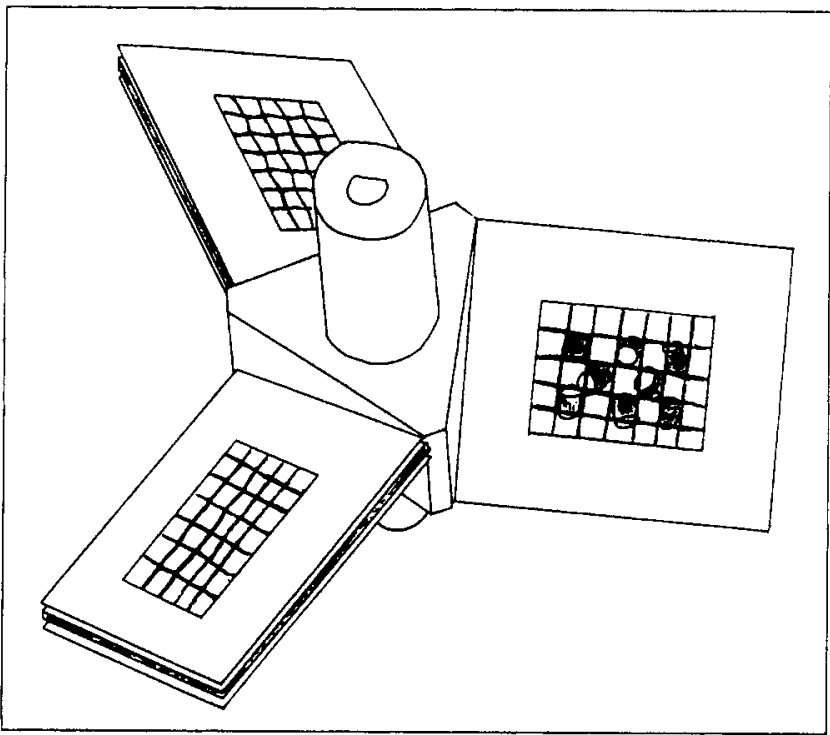

Figure 2. Axial flow impeller with rectangular openings in the blades in which particles are fixed be. tween gauzes.

\section{Reactor Principles}

In our reactor, perfectly mixed conditions are achieved by circulating the gas by means of an axial flow impeller in a well streamlined enclosure: the reactor has a rounded shape so as to guide the gas flow smoothly through the reactor and to prevent dead zones. To suppress a circular movement of the gas four baffles with a width of one-tenth of the total internal diameter are situated on the wall of the reactor. A schematic drawing of the reactor is given in Figure 1. The diameter of the reactor was chosen such that the cross-sectional area covered by the impeller was equal to that between the impeller and the reactor wall. Thus, compression and expansion of the gas is reduced. The catalyst particles are located inside a rectangular opening in the blades of the impeller, as shown in Figure 2. Figure 3 shows gas velocity components around the catalyst particle. The velocity of the particles in reactor is $v_{t}=2 \pi N r$, where $r$ is the distance of the particle to the shaft axis and $N$ is the rotational velocity. We will determine ex-

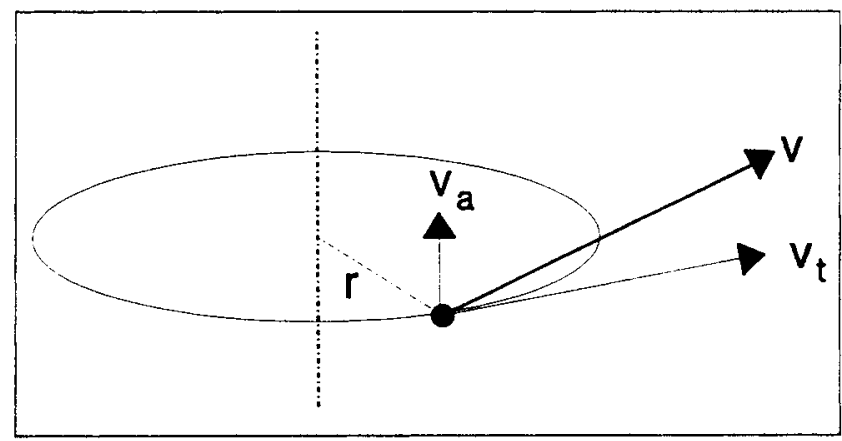

Figure 3. Gas velocity components around catalyst particle.

$v_{a}$ gas velocity of circulating flow, $v$, rotating velocity of particle, and $v$ total gas velocity. 
perimentally whether this velocity or the recycle flow rate controls the transport rates.

The degree of mixing in the reactor is determined by the impeller discharge rate, $Q$, given by:

$$
Q=N_{Q} N D^{3} \quad\left(\mathrm{~m}^{3} / \mathrm{s}\right)
$$

In this relation $D$ is the impeller diameter, $N$ the impeller speed, and $N_{Q}$ is a discharge constant or pumping coefficient, which for a pitched blade impeller at an impeller Reynolds number $D^{2} N \rho_{g} / \eta=10^{5}$ has a value of about 0.4 , as shown in Chapter 19 of Perry and Green (1984). A large impeller diameter was chosen as this increases the discharge rate. A direct consequence of the basic design is a large reactor volume. The large volume was chosen deliberately as in this design heat and mass transfer and mixing are facilitated. The large volume has no consequences when investigating truly heterogeneous reactions: reaction rates are not affected; these are only influenced by the mass-flow rate, the composition of the feed gas, and the catalyst mass in the reactor. The empty reactor volume only influences reactor dynamics.

\section{Detailed Design of the Reactor}

The impeller is placed in the center of the reactor and has, apart from the rectangular opening in each blade, the shape of a conventional pitched-blade impeller and a diameter of 130 $\mathrm{mm}$, as shown in Figure 2. It has three flat blades of $54 \times 38 \times 2$ $\mathrm{mm}$ at an angle of $30^{\circ}$ with the plane of rotation. In every blade there is a rectangular opening of $28 \times 20 \mathrm{~mm}$ in which catalyst particles can be fixed between two gauzes. The distance between the particles and the impeller axis varies from 30 to $50 \mathrm{~mm}$. The maximum rotational velocity is $50 \mathrm{~s}^{-1}$, resulting in a maximum particle velocity of $15.7 \mathrm{~m} / \mathrm{s}$. The total reactor volume equals 3.3 liters, as shown in Table 1 . The reactor consists of three parts: a top and a bottom part which are equipped with pressure sealed leads for thermocouples, and a center part in which the gas inlet and outlet are situated. The impeller has bearings both in the top and the bottom part of the reactor; thus imbalances, as occur with shafts with only one bearing, are avoided. The impeller motor is magnetically coupled to the shaft of the impeller. Both the reactor and the impeller are constructed from stainless steel 316 . The reactor is surrounded by a bronze jacket which prevents uneven heating and is placed in an electric oven with which the desired operating temperature is controlled. The prototype was designed for pressures up to 25 bar and temperatures up to $300^{\circ} \mathrm{C}$.

\section{Reactor Performance}

The performance of the reactor has been tested in several ways: We studied the interparticle mass-transfer rates and performed experiments under reacting conditions using the fast and strongly exothermic hydrogenation of ethene. No residence time distribution experiments were done because for our reactor at the shortest residence time, and the lowest rotational speed of the impeller for the estimated recycle ratio is still about 25 , so only perfect mixing will be found (Westerterp et al., 1987). The impeller was kept running for three months continuously during which no problems with the bearings of the shaft occurred.
Table 1. Reactor Dimensions and Design Characteristics

\begin{tabular}{lc}
\hline Characteristic & Dimension \\
\hline Reactor volume & $3.3 \mathrm{~L}$ \\
Maximum height & $0.16 \mathrm{~m}$ \\
Maximum width & $0.18 \mathrm{~m}$ \\
Design pressure & $20 \mathrm{bar}$ \\
Design temperature & $300^{\circ} \mathrm{C}$ \\
Number of baffles & 4 \\
Impeller diameter & $0.13 \mathrm{~m}$ \\
\hline
\end{tabular}

\section{Measurement of mass-transfer coefficients}

Particles Rotating with the Impeller. Mass-transfer coefficients were determined by measuring the evaporation of naphthalene pellets. To this end a number of cylindrical naphthalene pellets are weighed and mounted onto the impeller. The gas flow rate was chosen such that the average concentration of naphthalene in the gas during the experiment is kept below saturation, typically $\langle C\rangle \approx 0.25 C^{i}$, to minimize experimental errors. The length of an experiment was selected such that the decrease of the surface area for each pellet was smaller than $10 \%$. At the end of the experiment the reactor was disassembled, the pellets were weighed again, and the masstransfer coefficient for each pellet was calculated using the relations given in Appendix A. In every experiment, the values for the mass-transfer coefficients are obtained in triple by placing particles at the same position in the three impeller blades and weighing each particle individually. The spread between these replicate values was less than $3 \%$.

The influence of the following variables on the mass-transfer rate was evaluated:

- Particle diameter

- Gas density

- Particle velocity

- Physical properties of the bulk gas

Equilateral cylindrical pellets of $4.1 \times 4.1$ and $8 \times 8 \mathrm{~mm}$ were used. The gas density was varied by changing the pressure between 0.1 and 1.0 MPa, and by using different gases. The particle velocity was varied by placing pellets at different distances from the impeller axis and by changing the impeller speed. Nitrogen, hydrogen, and sulfur-hexa-fluoride were used as bulk gases. Table 2 summarizes the physical properties of the gases. The diffusion coefficients were estimated using the method of Fuller (Reid et al., 1987). The experimental results are shown in Figure 4. All experiments could be correlated with the following relation:

$$
S h=0.3+0.07 R e^{0.76} S c^{0.54}
$$

The value of the Sherwood number at $R e=0$ was not deter-

Table 2. Physical Properties of Dilute Naphthalene Gas Mixtures at $300 \mathrm{~K}$ and $0.1 \mathrm{MPa}$

\begin{tabular}{lcccc}
\hline Gas & $\begin{array}{c}\text { Diffusion } \\
\text { Coefficient } \\
\left(\mathrm{m}^{2} / \mathrm{s}\right)\end{array}$ & $\begin{array}{c}\text { Viscosity } \\
(\mathrm{Pa} \cdot \mathrm{s})\end{array}$ & $\begin{array}{c}\text { Density } \\
\left(\mathrm{kg} / \mathrm{m}^{3}\right)\end{array}$ & $\begin{array}{c}\text { Schmidt } \\
\text { No. for }\end{array}$ \\
\hline $\mathrm{H}_{2}$ & $31.9 \times 10^{-6}$ & $8.86 \times 10^{-6}$ & 0.0823 & 3.3 \\
$\mathrm{~N}_{2}$ & $7.5 \times 10^{-6}$ & $17.7 \times 10^{-6}$ & 1.13 & 2.1 \\
$\mathrm{SF}_{6}$ & $2.7 \times 10^{-6}$ & $15.2 \times 10^{-6}$ & 5.9 & 1.0 \\
\hline
\end{tabular}




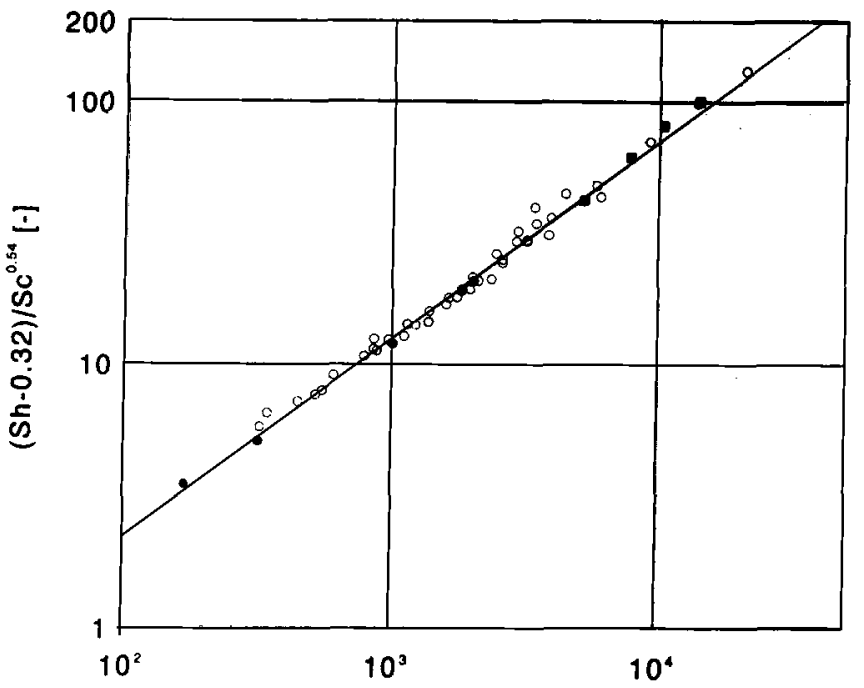

Re [-]

Figure 4. Correlation for the rate of mass transfer of pellets moving with the impeller.

$(S h-0.3) / S c^{0.54}$ as function of the Reynolds number. $0: \mathrm{N}_{2} ; \bullet$ : $\mathrm{H}_{3} ; \mathbf{0}: \mathrm{SF}_{6}$. The solid line is given by Eq. $2 . \mathrm{Sc}^{0.54}$ equals 1.9 , 1.5 , and 1.0 for $\mathrm{H}_{2}, \mathrm{~N}_{2}$, and $\mathrm{SF}_{6}$, respectively.

mined but fixed at the empirical value 0.3 for cylinders ( $\operatorname{Ranz}$, 1952). The exact value of this constant is not of major importance as the experimental Sherwood numbers are much higher than 0.3. The other constant and the exponents in relation 2 were fitted to the experimental data. In the relation, the Reynolds number is based on the equivalent sphere diameter and the particle velocity $v_{t}$. Mass-transfer coefficients, obtained at equal impeller speeds and for particles placed at different distances from the impeller axis, can be uniquely correlated using relation 2, as shown in Figure 5. It can also be demonstrated that the influence of the circulating flow rate on the mass-transfer coefficients is negligible. The discharge

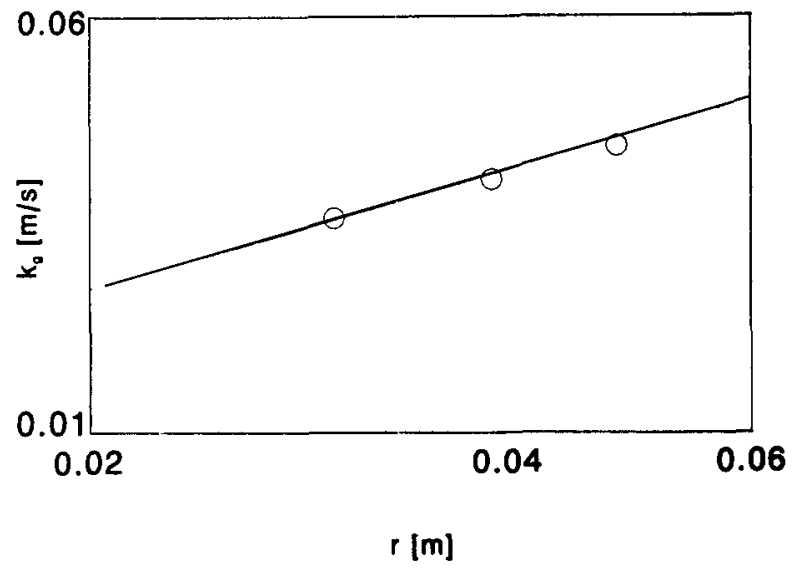

Figure 5. Mass-transfer coefficient for pellets moving with the impeller located at different distances from the impeller axis.

Experiment with nitrogen; $P=0.1 \mathrm{MPa} ; T=294 \mathrm{~K} ; N=12.0 \mathrm{~s}^{-1}$; the solid line is given by Eq. 2 .

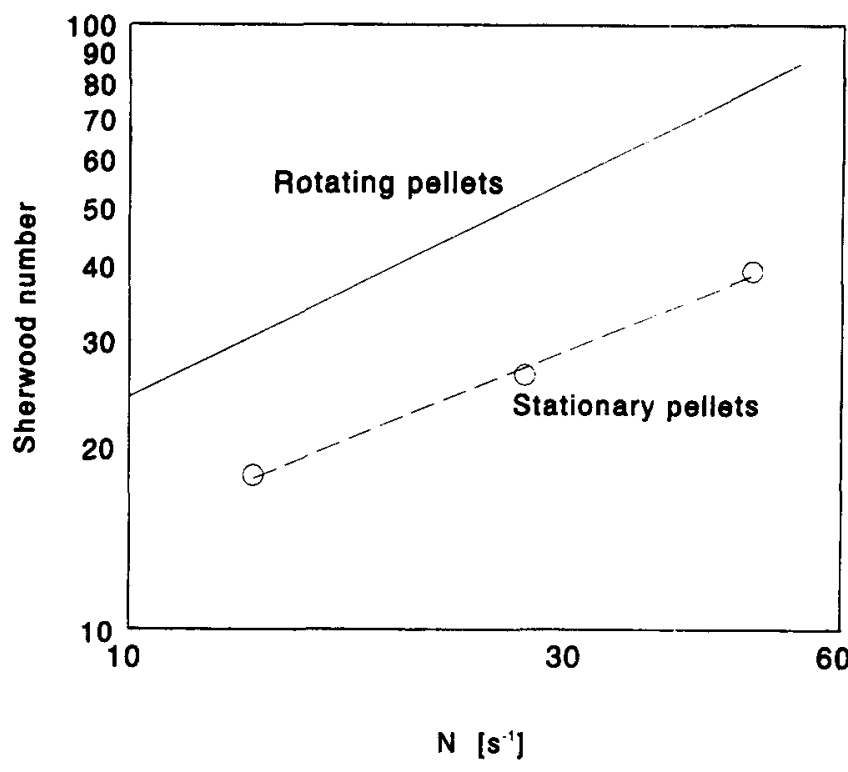

Figure 6. Comparison of mass-transfer rates for pellets moving with the impeller and for a pellet fixed on a wire in the reactor.

$T=293 \mathrm{~K}$ and $P=0.1 \mathrm{MPa}$; the solid line is calculated from Eq. 2 for a pellet of $d_{p}=8 \mathrm{~mm}$ at a distance of $4 \mathrm{~cm}$ from the impeller axis; the measured data were obtained for a pellet of $d_{p}=8 \mathrm{~mm}$ fixed on a wire.

rate of the impeller can be estimated using Eq. 1. For the mean axial velocity, the gas velocity perpendicular to the plane of rotation of the impeller-assuming a value of 0.4 for the discharge coefficient-is:

$$
v_{a}=\frac{Q}{\frac{1}{4} \pi D^{2}}=\frac{4 N_{Q} N D^{3}}{\pi D^{2}} \approx 0.5 N D
$$

The impeller thus moves a quantity of gas through the plane of rotation in the axial direction while the particles located on the impeller rotate tangentially with a velocity $v_{t}$. If we suppose as a worst case that both velocity contributions are additive we find for the ratio between the actual velocity, $v$, and the particle velocity, $v_{t}$, as shown in Figure 3:

$$
\frac{|v|}{\left|v_{t}\right|}=\sqrt{1+\frac{\left|v_{a}\right|^{2}}{\left|v_{t}\right|^{2}}}=\sqrt{1+\left[\frac{0.5 N D}{2 \pi N r}\right]^{2}} \approx \sqrt{1+\left[\frac{1}{12} \frac{D}{r}\right]^{2}}
$$

For our impeller the maximum value for $D / r$ is 4.3 which results in 1.06 for $|v| /\left|v_{r}\right|$, so the axial velocity component can be neglected. The particle velocity, $v_{t}$, is approximately three times higher than the mean axial velocity $v_{a}$, as can be calculated from Eqs. 1 and 4.

From Figure 4 it can also be seen the influence of pressure, particle diameter, and physical properties of the gas on the mass-transfer coefficient are well represented by relation 2 .

Particles Fixed on a Wire in the Reactor. In a number of experiments mass-transfer coefficients were determined for naphthalene particles in a fixed position in the reactor, and these particles do not move with the impeller. A particle was fixed by drilling a hole in it and then placing it on a ther- 


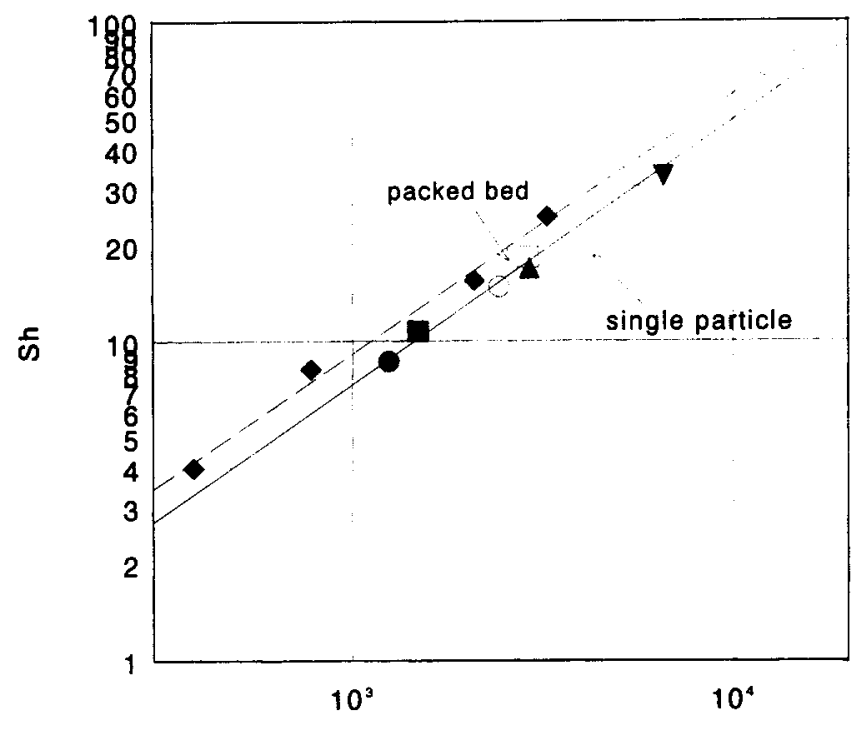

$\mathrm{Re}$,

Figure 7. Mass-transfer experiments in a Berty reactor: Sherwood number as function of a modified particle Reynolds number.

Bulk gas: nitrogen; closed symbols: $d_{p}=4.1 \mathrm{~mm}$; open symbols: $d_{\rho}=8 \mathrm{~mm} ; \circ: 0.28 \mathrm{MPa}$ 口: $0.53 \mathrm{MPa} ; \Delta: 1.01 \mathrm{MPa} ; \nabla: 1.03$ MPa for experiments with single particles. experiments with pellets in a packed bed of inert particles.

mocouple, as shown in Figure 1. The results of these experiments are shown in Figure 6. At equal operating conditions, the mass-transfer coefficient for a fixed particle is a factor of 2 lower than for a particle rotating with the impeller. This means the local gas velocity around the fixed particle is a factor $2^{1 / 0.8}=2.4$ lower, as the mass-transfer coefficient is proportional to the gas velocity to the power 0.8 , see Eq. 2 . This value corresponds reasonably with the estimate of the mean axial velocity as given in the previous section.

Mass Transfer in an Internal Recycle Reactor. To compare the mass-transfer rates in our reactor to those in an internal recycle reactor also mass-transfer experiments were performed in a commercial Berty reactor (Berty, 1974). A detailed description of the reactor in which the experiments were performed has been presented by Bos et al. (1992). In this reactor, the mass-transfer rate is determined by the recycle flow rate. The Sherwood number will be a function of the particle Reynolds number, $\rho_{g} v_{g} d_{p} / \eta_{g}$, and the physical properties of the gas. The mean gas velocity, $v_{g}$, is proportional to:

$$
v_{g}=\frac{Q}{A}=\frac{N_{Q} N D^{3}}{\operatorname{const} \frac{1}{4} \pi D^{2}} \propto N D,
$$

where $A$ is the cross-sectional area of the basket in which the particles are placed. The diameter of this cross section is proportional to that of the impeller. A modified particle Reynolds number can be defined as:

$$
R e_{p}^{*} \equiv \frac{\rho_{g} N D d_{p}}{\eta}
$$

In Figure 7 experimental results are presented for pellets of $4.1 \times 4.1$ and $8 \times 8 \mathrm{~mm}$ at pressures between 0.28 and 1.03 $\mathrm{MPa}$, and at varying impeller speeds. The bulk gas was nitrogen. In each experiment, only one small and one large pellet were placed in the Berty-reactor. All data could be uniquely correlated as:

$$
S h_{s p}=0.024\left(R e_{p}^{*}\right)^{0.83}
$$

When the basket is filled with more particles the recycle flow rate will decrease as the resistance against flow increases, and therefore the mass-transfer coefficients decrease. In a packed bed, the mass-transfer coefficients are higher than those for single particles. An equation relating Sherwood numbers for cylinders in a packed bed to those for single particles is given by Gnielinski (1982):

$$
S h_{p b}=1.6 S h_{s p}
$$

At equal superficial velocities, the single particle Sherwood numbers in a packed bed are also higher when compared to a true single particle because of the higher interstitial gas velocities. The overall enhancement of the mass-transfer coefficient for cylinders in a packed bed compared to a single cylinder in a gas stream at equal superficial velocities is a factor $1.6(1 /$ $\epsilon)^{0.8}$, so between 2.5 and 3 approximately.

In Figure 7 results are also shown of mass-transfer experiments with naphthalene pellets in a bed packed with inert alumina pellets of $4 \times 4 \mathrm{~mm}$. The bed had a diameter of 3.5 $\mathrm{cm}$, a height of $1 \mathrm{~cm}$, and an estimated porosity of 0.6 . The mass-transfer data for these packed bed experiments were correlated as:

$$
S h_{p b}=0.033\left(R e_{p}^{*}\right)^{0.81}
$$

The packed bed Sherwood numbers are $40 \%$ higher than those determined for single particles at equal operating conditions and not 2.5 to 3 times, which would be expected for equal superficial gas velocities. From the change in the Sherwood numbers, the variation of the superficial gas velocity in the "empty" reactor is estimated to be 2.3 times as high compared to that in the reactor with the packed bed. We see that even the small packed bed used here with a height of only two particles layers leads to a drastic decrease of the recycle flow rate.

In Figure 8, the Sherwood number for a single pellet of $4.1 \times 4.1 \mathrm{~mm}$ in the Berty reactor is compared to that for a pellet located on the impeller in the novel reactor. At equal tip velocities of the impeller $N D$, we find that the mass-transfer rate in the new reactor is 4 to 6 times higher. The impeller in the Berty reactor had a diameter of $7.5 \mathrm{~cm}$. In our reactor, the maximum impeller speed is $50 \mathrm{~s}^{-1}$, and that in the recycle reactor is $30 \mathrm{~s}^{-1}$. Considering the better balancing of the shaft in the new reactor, it allows for much higher rotational speeds and tip velocities. Thus, in the novel reactor mass-transfer rates can be realized at least a factor of 10 higher than in our recycle reactor.

\section{Operation with a fast reaction}

The reactor was tested using the hydrogenation of ethene 


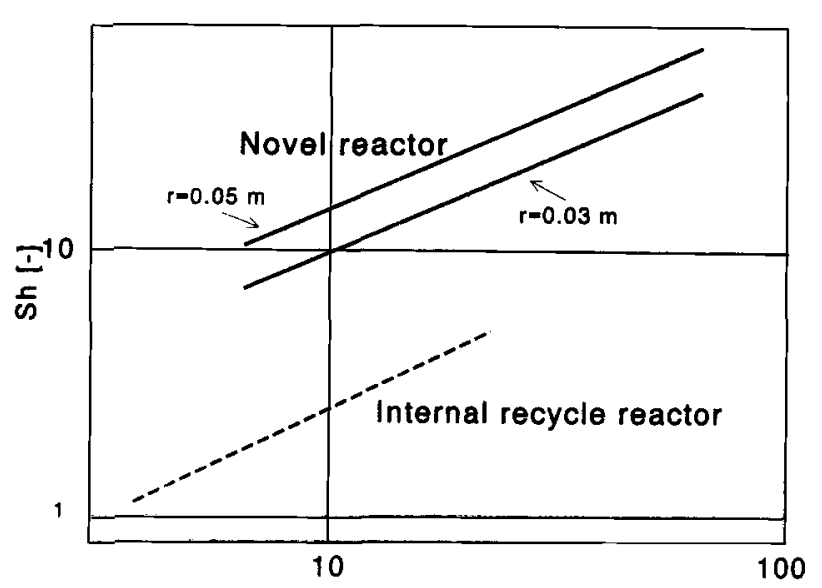

ND $[\mathrm{m} / \mathrm{s}]$

Figure 8. Mass-transfer coefficients in an internal recycle reactor compared to those in the novel reactor.

The Sherwood number for a particle of $d_{p}=4.1 \mathrm{~mm}$ as function of the tip velocity of the impeller using nitrogen as bulk gas at $P=0.1 \mathrm{MPa}$. The lines start and end at respectively the minimum and the maximum possible tip velocities which could be realized in our setups.

over Palladium supported on a $\gamma$-alumina catalyst of the eggshell type. This reaction is fast and strongly exothermic:

$$
\mathrm{C}_{2} \mathrm{H}_{4}+\mathrm{H}_{2} \rightarrow \mathrm{C}_{2} \mathrm{H}_{6} \quad \Delta \mathrm{H}=-137 \mathrm{~kJ} / \mathrm{mol}
$$

In a previous kinetic study in an internal recycle reactor, the observed rate was found to be severely influenced by transport resistances (Bos et al., 1992). In our experiments, 45 cylindrical pellets with a height and a diameter of $4.4 \mathrm{~mm}$ were located on the impeller. In the experiments, also one single catalyst particle was fixed on a stationary thermocouple.

This particle does not move with the impeller. The temperature difference between this pellet and the gas is an indication for the influence of transport resistances on reaction rates for this pellet. Temperature differences between the gas and pellets rotating with the impeller-which we are actually interested in-cannot be measured in our setup. Thus, the values for the particles fixed on a thermocouple serve as a worst case estimate. Temperature differences between gas and particles moving with the impeller will be smaller as transport rates for these pellets are higher, as was shown in the previous section.

In Figure 9a, a situation is shown where upon decreasing the rotational speed of the impeller the temperature difference between the particle fixed on the thermocouple and the gas increases, whereas the total reaction rate as observed for all pellets is not significantly affected. Thus, we see that the reaction rate for the fixed particle is influenced by transport resistances, whereas that for the particles moving with the impeller is not. In Figure 9b, a case is shown where at low impeller speeds also the observed rate of reaction is influenced by the impeller speed. At these low impeller speeds also, the reaction rate for the pellets moving with the impeller is influenced by transport resistances. The experimental results in Figure 9 teach us that in our reactor the temperature difference

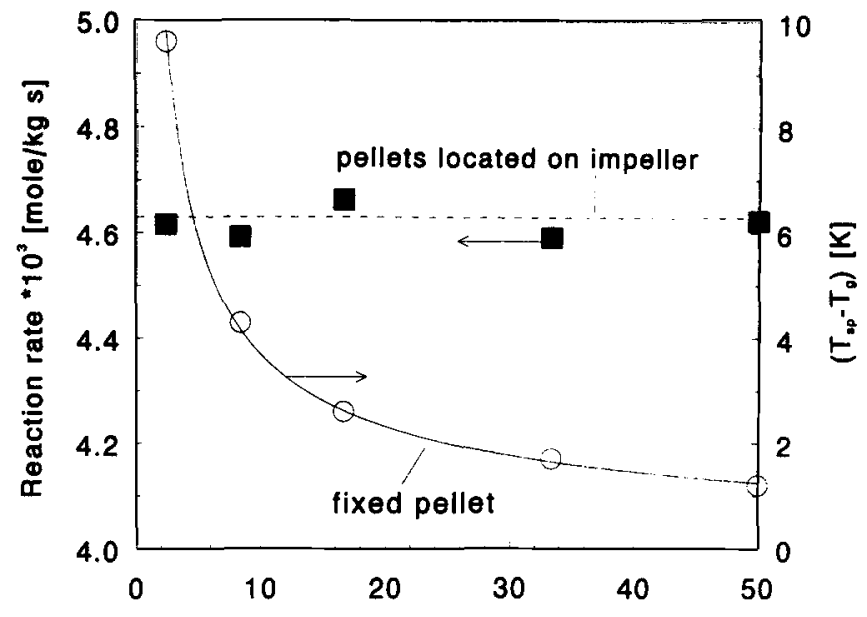

Impeller speed $\left[\mathrm{s}^{-1}\right]$

(a)

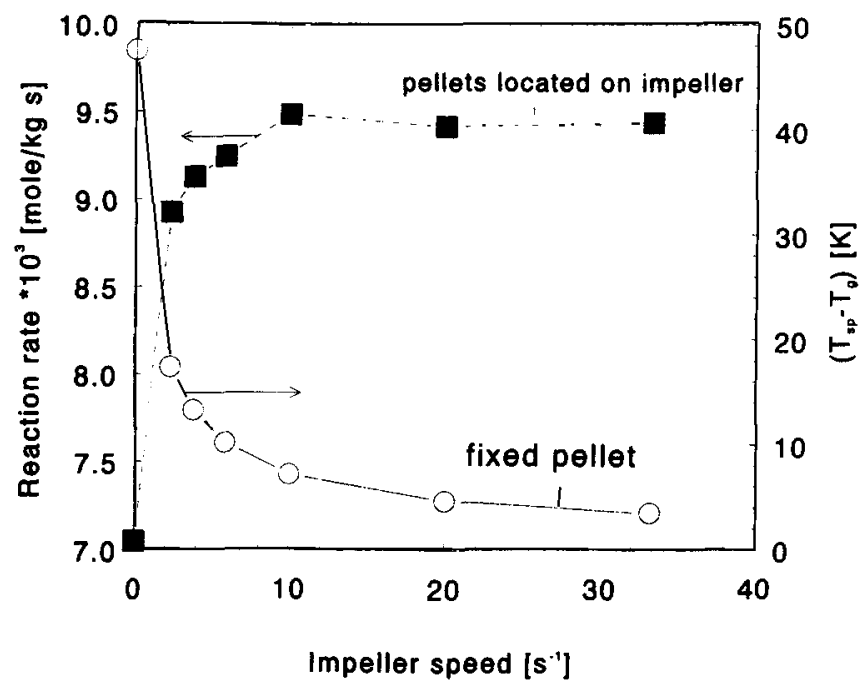

(b)

Figure 9. Influence of impeller speed on ethene hydro. genation rates and the temperature difference between the gas and a catalyst particle fixed on thermocouple in the reactor, this particle is not moving with the impeller.

(a) Experiment at $P=.13 \mathrm{MPa} ; T_{g}=37^{\circ} \mathrm{C}$; feed flow $4.9 * 10^{-3}$ $\mathrm{mol} / \mathrm{s}$ and a hydrogen mole fraction in the feed of $4.85 \%$; (b) Experiment at $P=0.16 \mathrm{MPa}, T_{g}=50^{\circ} \mathrm{C}$, feed flow $8.9 \times 10^{-4} \mathrm{~mol} /$ $\mathrm{s}$, hydrogen mole fraction in feed $5.3 \%$.

between gas and a stationary single particle gives far too conservative information on transport limitations. Therefore, a test at different impeller speeds must be used to give the final clue on the absence of transport limitations.

\section{Discussion and Conclusions}

Of course, there are some limitations to the novel reactor design. There is a lower bound to the size of catalyst particles which can be placed in the impeller; in our case, only catalyst particles with a diameter exceeding $2 \mathrm{~mm}$ can be used. The 
centrifugal force exerted on the catalyst particles could damage them. In our design, the volume of the reactor not filled with catalyst is large. This empty volume is important when homogeneous reactions occur; in this case, it should be kept as small as possible. The residence time of the gas in the reactor and therefore also the time to reach a steady state is long, especially when operating at high pressures. This is a disadvantage for steady-state studies. However, the slow dynamics of the reactor could be advantageous for determining steadystate kinetics from dynamic experiments. The high mass of the prototype reactor, approximately $30 \mathrm{~kg}$, causes a long heatup time. At the same time, this large mass facilitates in maintaining the reactor isothermal at reaction conditions.

A perfectly mixed laboratory reactor for determining kinetics of solid catalyzed gas reactions has been developed in which a better degree of mixing as well as much higher interparticle mass- and heat-transfer rates can be achieved compared to the presently available reactor types.

From mass-transfer experiments, it was shown that heatand mass-transfer rates between a particle and the gas are determined by the linear velocity of that particle in the reactor. The degree of mixing in the reactor is determined by the circulating flow rate. Thus, interparticle transport and mixing depend on different phenomena. In an internal recycle reactor, both depend on the recycle flow rate. In the new reactor, the recycle flow rate is independent of the total amount of catalyst contained in the reactor, whereas in an internal recycle reactor it strongly depends on the resistance to flow posed by the catalyst bed.

\section{Acknowledgment}

The authors would like to thank Dr. I. Racz for advice on the design of the reactor and P. A. Dieperink and F. L. Luikinga for performing the experimental work.

\section{Notation}

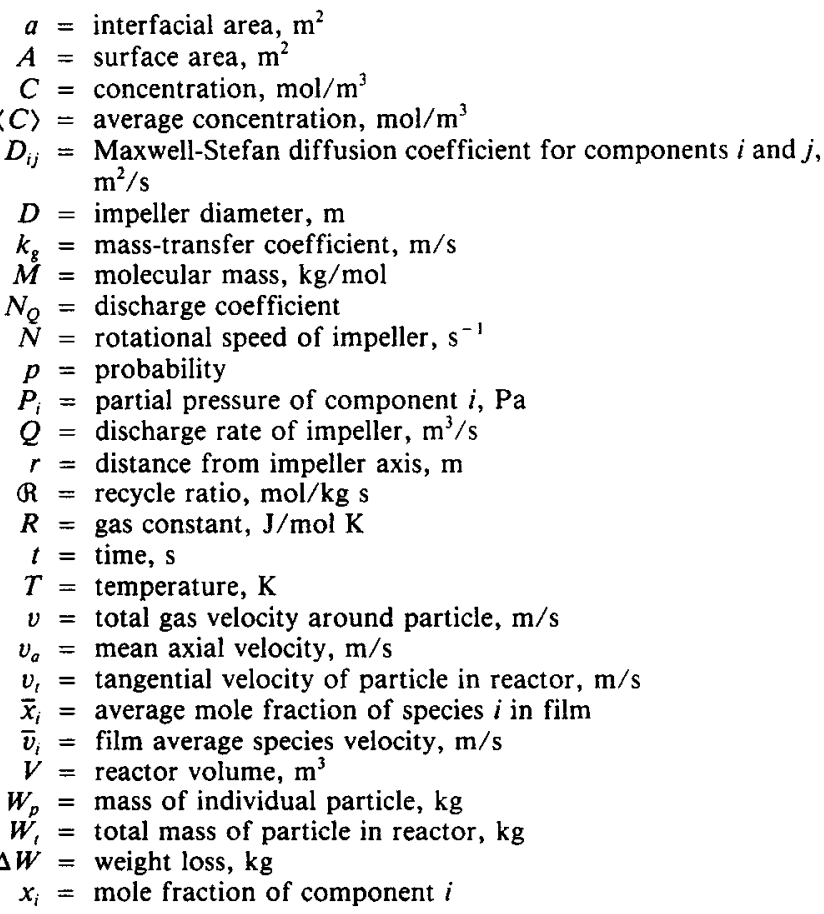

\section{Greek letters}

$\delta=$ mass-transfer film thickness, $\mathrm{m}$

$\eta=$ viscosity, $\mathrm{Pa} \cdot \mathrm{s}$

$\mathrm{N}=$ molar flux of species $i, \mathrm{~mol} /\left(\mathrm{m}^{2} \cdot \mathrm{s}\right)$

$\Xi=$ criterion defined by Eq. All

$\rho=$ density, $\mathrm{kg} / \mathrm{m}^{3}$

$\tau_{c}=$ circulation time, $\mathrm{s}$

$\tau_{r}=$ residence time, $\mathrm{s}$

$\phi=$ feed mass flow, $\mathrm{kg} / \mathrm{s}$

\section{Subscripts and superscripts}

$$
\begin{aligned}
a c & =\text { accumulated } \\
c & =\text { circulating } \\
e & =\text { end of experiment } \\
g & =\text { gas phase } \\
i & =\text { interface } \\
p & =\text { particle } \\
p b & =\text { packed bed } \\
s & =\text { solid } \\
s p & =\text { single particle } \\
t & =\text { total } \\
1 & =\text { naphthalene } \\
2 & =\text { bulk gas }
\end{aligned}
$$

\section{Dimensionless groups}

$S h \equiv\left(k_{g} d_{p}\right) / D_{i, j}$, Sherwood number

$R e \equiv\left(\rho_{g} v, d_{p}\right) / \eta_{g}$, particle Reynolds number based on the tangential velocity of the particle

$R e_{p}^{*} \equiv\left(\rho_{g} N D d_{p}\right) / \eta_{g}$, modified particle Reynolds number

$S c \equiv \eta_{g} /\left(\rho_{g} D_{i, j}\right)$, Schmidt number

\section{Literature Cited}

Berty, J. M., "Reactor for Vapour-Phase Catalytic Studies," Chem. Eng. Prog., 70, 78 (1974).

Berty, J. M., "Testing Commercial Catalysts in Recycle Reactors," Catal. Rev.-Sci. Eng., 20, 75 (1979).

Berty, J. M., "20 Years of Recycle Reactors in Reaction Engineering," Plant. Op. Prog., 3, 163 (1984).

Borman, P. C., "Falsification of Experimental Kinetic Data Obtained in a Recycle Reactor," The Selective Oxidation of Ethene in a Wall Cooled Tubular Packed Bed Reactor, Thesis, Twente Univ., Enschede (1992).

Bos, A. N. R., E. S. Bootsma, F. Foeth, H. W. J. Sleyster, and K. R. Westerterp, "A Kinetic Study of the Hydrogenation of Ethyne and Ethene on a Commercial $\mathrm{Pd} / \mathrm{Al}_{2} \mathrm{O}_{3}$ Catalyst," Chem. Eng. Proc., 32, 53 (1993).

Broucek, R., "Falsification of Kinetic Parameters by Incorrect Treatment of Recirculation Reactor Data," Chem. Eng. Sci., 38, 1349 (1983).

Carberry, J. J., "Designing Laboratory Catalytic Reactors," Ind. Eng. Chem., 56, 39 (1964).

Christoffel, E. G., "Laboratory Reactors and Heterogeneous Catalytic Processes," Catal. Rev.-Sci. Eng., 24, 159 (1982).

Daubert, T. E., and R. P. Danner, Physical and Thermodynamic Properties of Pure Chemicals, Data Compilation, Hemisphere, New York (1984)

Doraiswamy, L. K., and D. G. Tajbl, "Laboratory Kinetic Reactors," Cat. Rev.-Sci. Eng., 10, 177 (1974).

Dümpelmann, R., and A. Baiker, "Criteria for Gradientless Operation of Internal Recycle Reactors," Chem. Eng. Sci., 47, 2665 (1992).

Georgakopoulos, K., and R. Broucek, "Investigation of the NonIdeality of an Internal Recycle Reactor Using a Concentration Controlled Test Reaction," Chem. Eng. Sci., 42, 2782 (1987).

Gnielinski, V., "Berechnung des Warme und Stoffaustauschs in durchstromten ruhenden Schuttungen," vt verfahrenstechn., 16, 36 (1982).

Perry, R. H., and D. Green, eds., Perry's Chemical Engineers' Handbook, 6th ed., McGraw-Hill, Singapore (1984).

Pirjamali, M., H. Livberg, and J. Villadsen, "A Correction Procedure 
for Interparticle Gradients in a Laboratory Catalytic Recirculation Reactor," Chem. Eng. Sci, 28, 328 (1973).

Ranz, W. E., "Friction and Transfer Coefficients for Single Particles and Packed Beds," Chem. Eng. Prog., 48, 247 (1952).

Reid, R. C., J. M. Prausznitz, and B. E. Poling, The Properties of Gases and Liquids, 4th ed., McGraw-Hill, New York (1987).

Wedel, S., and J. Villadsen, "Falsification of Kinetic Parameters by Incorrect Treatment of Recirculation Reactor Data," Chem. Eng. Sci. 38, 1346 (1982).

Weekman, V. W., "Laboratory Reactors and their Limitations," AIChE J., 20, 833 (1974).

Wesselingh, J. A., and R. Krishna, Mass Transfer, Ellis Horwood, Chichester (1990).

Westerterp, K. R., W. P. M. van Swaaij, and A. A. C. M. Beenackers, Chemical Reactor Design and Operation, Wiley (1987).

\section{Appendix: Experimental Determination of Mass- Transfer Coefficients}

For the evaporation of naphthalene the equation for the flux, $N_{1}$, to be used in the mass balance equations can be derived from the difference form of the Maxwell-Stefan transport equation for this binary problem (Wesselingh and Krishna, 1990):

$$
\frac{\Delta x_{1}}{\overline{x_{1}}}=\overline{x_{2}} \frac{\overline{v_{2}}-\overline{v_{1}}}{\frac{D_{12}}{\delta}}
$$

With the bootstrap relation:

$$
\overline{v_{2}}==0
$$

for the flux of naphthalene it follows:

$$
\mathrm{N}_{1}=-C_{t} \overline{x_{1}} \overline{v_{1}}=-C_{t} \frac{D_{12}}{\delta} \frac{\Delta x_{1}}{1-\overline{x_{1}}}
$$

The heat effect of the evaporation can be neglected. $\overline{x_{2}}=1-\overline{x_{1}} \approx 1$ because of the low partial pressure of naphthalene at the experimental conditions. Thus, the usual Fick relation can be used:

$$
\mathrm{N}_{1}=-k_{\mathrm{g}} \Delta C_{1}
$$

The mass balance for a single particle is:

$$
\frac{1}{M_{1}} \frac{d W_{p}}{d t}=k_{g} a_{p}\left(C_{1}^{i}-C_{1}^{g}\right)
$$

Integration of the differential equation, assuming the particle surface area to be constant, yields:

$$
\Delta W_{\rho}=k_{g} a_{p} M_{1}\left[C_{l}^{i} t_{e}-\int_{t=0}^{t_{e}} C_{l}^{g}(t) d t\right]
$$

or

$$
\frac{\Delta W_{p}}{t_{e}}=k_{g} a_{p} M\left(C_{1}^{i}-\left\langle C_{1}\right\rangle\right)
$$

In this relation $\left\langle C_{1}\right\rangle$ is the average naphthalene concentration in the gas phase during the experiment. It can be estimated by assuming that all evaporated naphthalene has left the reactor in the effluent gas at the conclusion of the experiment:

$$
\left\langle C_{1}\right\rangle \equiv \frac{\int_{t=0}^{t_{e}} C_{1}^{g}(t) d t}{t_{e}} \approx \frac{\rho_{g} \Delta W_{t}}{M_{1} \phi t_{e}} \quad\left(\mathrm{~mol} / \mathrm{m}^{3}\right)
$$

Not correcting the driving force for partial saturation of the gas phase causes an underestimation of the mass-transfer coefficients. In the experiments, $\left\langle C_{1}\right\rangle / C_{i}^{i}$ was typically $25 \%$.

During an experiment an amount of naphthalene, $W_{a c}$, will accumulate in the gas phase of the reactor. This amount is equal to:

$$
W_{a c} \equiv M_{1} V\left(C_{1}^{t=t_{e}}-C_{1}^{l=0}\right) \quad(\mathrm{kg})
$$

and smaller than:

$$
W_{a c} \leq M_{1} V C_{1}^{\prime} \quad(\mathrm{kg})
$$

The error introduced by applying Eq. A8 will be acceptable when $\Delta W_{l} \gg W_{a c}$ or

$$
\Xi=\frac{\Delta W_{t}}{M_{1} V C_{1}^{i}} \gg 1
$$

The value of $\Xi$ can be manipulated by increasing the number of naphthalene pellets in the reactor, or increasing the length of an experiment, both leading to an increase of $\Delta W_{t}$. If $\Xi$ has too low a value, Eq. A8 will predict too high a value of $\left\langle C_{1}\right\rangle$, leading to an overestimation of the mass-transfer coefficient. In our experiments, $\Xi$ typically exceeded 10 . The concentrations of naphthalene at the interface were calculated assuming the gas to be ideal:

$$
C_{1}^{i}=\frac{P_{1}}{R T}
$$

Daubert and Danner (1984) give the following relation for the vapor pressure of naphthalene:

$$
\ln \left(P_{1}\right)=22.817-\frac{8,609.3}{T}+1.768 \ln (T)-5.429510^{-3} T
$$

When the weight loss of a naphthalene particle after a period of $t_{\mathrm{e}}$ is measured the mass-transfer coefficient for this particle can be calculated using relations A7, A8, A11 and A12. 